\title{
Smoking, psychiatric illness and the brain
}

\author{
Patricia Boksa, PhD
}

Tobacco smoking remains a leading cause of mortality and morbidity despite recent declines in prevalence. In 2012, global prevalence rates for tobacco smoking were estimated to be $31 \%$ for men and $6 \%$ for women. ${ }^{1}$ Rates of smoking are markedly higher among people with psychiatric illness than in the general population, estimated at being 2-5 times higher in patients with several disorders, including schizophrenia, mood disorders, anxiety disorders, attentiondeficit/hyperactivity disorder (ADHD), binge eating disorder, bulimia and substance use disorders. ${ }^{2-5}$

The aim of this editorial is to emphasize the importance of smoking as a factor that should regularly be considered as a potential confound in neurobiological studies of psychiatric illnesses. Smoking can be regarded as playing at least several roles in relation to psychiatric illness - as a causal factor contributing to psychiatric disorders, as an agent causing brain changes on its own that may interact with psychiatric pathophysiology, or as a modulator of effects of psychotropic medications. In each of these roles, smoking may impact the course of psychiatric illness and brain function.

\section{Smoking as a causal factor for psychiatric disorders}

There is strong evidence for an association between smoking and the development or progression of psychiatric disorders at the 2 ends of the developmental timeline - namely, ADHD and Alzheimer disease/dementia. In the case of ADHD, a large body of evidence supports an association between maternal smoking during pregnancy and increased risk for ADHD. ${ }^{5}$ However, whether maternal smoking is actually a causal factor for ADHD remains open to question, with recent research suggesting that the association may be due to a shared familial/genetic susceptibility for both smoking and ADHD. ${ }^{5}$ Nonetheless, children with ADHD born from mothers who smoked during pregnancy have been reported to have more severe behavioural problems than children with ADHD born from nonsmoking mothers, with a dose-response relationship between the amount smoked and several cognitive and clinical variables in the children. ${ }^{6}$ At a neurobiological level maternal smoking during pregnancy has been shown to be associated with grey matter loss and cortical thinning ${ }^{7}$ as well as functional alteration in neural circuitry involved in inhibitory control and reward ${ }^{8,9}$ in children.

There is also strong epidemiological evidence that smoking is associated with increased risk for dementia and the development of Alzheimer disease..$^{10}$ In a 2-year longitudinal study of 186 healthy elderly individuals, Durazzo and colleagues ${ }^{11}$ found that the rate of atrophy in several brain regions known to be affected by Alzheimer disease was greater in those with a history of smoking than in never smokers. ${ }^{11}$ In a similar vein, in a study including elderly individuals with mild cognitive impairment or Alzheimer dementia and controls, Tiepel and colleagues ${ }^{12}$ recently reported that smoking was associated with smaller basal forebrain volume in elderly patients with mild cognitive impairment as well as in healthy elderly individuals, suggesting that smoking may increase risk for Alzheimer disease by compromising cholinergic reserve capacity. ${ }^{12}$ Although the precise mechanism by which smoking produces central nervous system (CNS) atrophy has not been pinpointed, it is plausible that these effects may be mediated through the burden of cardiovascular impairment caused by smoking and by the cumulative direct cytotoxic effects of some of the thousands of compounds present in cigarette smoke. ${ }^{11}$

Although much more highly speculative at this point, a contributory role for smoking in the pathogenesis of other psychiatric disorders has been postulated. For example, it has been suggested that smoking may contribute to the development and maintenance of anxiety disorders through nicotine's modulation of fear memory and emotion processing. ${ }^{13}$ Effects of smoking on monoaminergic and glutamatergic systems, oxidative stress, and inflammatory and neurotrophic processes have also been hypothesized to contribute to the neuropathological mechanisms involved in the progression of bipolar disorder. ${ }^{14}$

\section{Smoking causes brain changes on its own that may affect psychiatric symptoms}

With regards to neuropsychological function, short-term administration of nicotine has positive effects on aspects of cognition, including learning, memory and attention in healthy

Correspondence to: P. Boksa, Douglas Mental Health University Institute, McGill University, 6875 LaSalle Blvd, Montreal, Que.; patricia.boksa@mcgill.ca

DOI: 10.1503/jpn.170060 
individuals, ${ }^{15-17}$ as well as in patients with schizophrenia, Alzheimer disease or ADHD. ${ }^{15,16}$ In contrast, chronic tobacco smoking has been shown to be associated with deficits in cognitive function, prominently verbal memory and processing speed, in middle-aged to elderly adults. ${ }^{16,17}$

Structural neuroimaging studies indicate that chronic tobacco smoking is associated with cortical thinning and size decreases in various brain structures. Karama and colleagues ${ }^{18}$ analyzed MRI data from 504 individuals at 73 years of age and reported diffuse accelerated cortical thinning, especially in prefrontal areas, in smokers compared with nonsmokers, and dose-dependent correlations between the amount smoked and the extent of cortical thinning in some regions. In a recent meta-analysis of structural MRI data, Sutherland and colleagues ${ }^{19}$ reported smoking to be associated with structural decreases in the insula, cerebellum, parahippocampus, prefrontal cortex and thalamus. Structural alterations, including decreases in grey matter volume of various brain structures and increased fractional anisotropy in diffusion tensor imaging studies, have been found not only in older smokers, but also in smokers younger than 30 years of age. ${ }^{20,21}$

At the level of functional neuroimaging, resting-state functional MRI (fMRI) studies of relatively large samples of smokers versus nonsmoking controls have reported altered connectivity in default and executive networks in the smoking groups. ${ }^{22,23}$ Task-related fMRI studies in smokers have often focused on neural circuitry thought to be involved in mediating addiction to nicotine. For example, such studies have shown alteration in function of neural circuitry involved in inhibitory control, attentional systems and risky decisionmaking in smokers compared with nonsmoking controls. ${ }^{24-26}$

Overall, there is now substantial evidence that tobacco smoking is associated with changes in brain structure and neural circuitry in brain regions and systems clearly implicated in many psychiatric disorders. These findings, together with the high prevalence of smoking in psychiatric populations, highlight the importance of including smoking as a potential confounding variable in studies examining neural mechanisms of psychiatric disorders.

\section{Smoking as a modulator of effects of psychotropic medications}

In addition to effects on its own, smoking/nicotine may modulate the metabolism of other psychoactive agents. Smoking is known to induce hepatic cytochrome P450 drugmetabolizing enzymes, particularly CYP1A2, involved in the metabolism of psychotropic drugs, including the antipsychotics clozapine and olanzapine, and other agents, such as other antipsychotics and benzodiazepines. ${ }^{27}$ Mean plasma levels of clozapine are lower in smokers than nonsmokers, and in a patient quitting smoking, clozapine levels are predicted to rise within 1-2 days after smoking cessation. ${ }^{27,28}$ CYP P450 enzymes are known to be present not only in peripheral organs, but also in the brain, where they may modulate local levels of endogenous and exogenous psychoactive compounds in a region-specific manner. ${ }^{29}$ For example, in various brain regions, smokers have higher levels of
CYP2B6, an enzyme capable of metabolizing endogenous compounds, such as serotonin and testosterone, as well as exogenous agents, such as nicotine, cocaine, amphetamines and buproprion. ${ }^{30}$ Therefore, in a number of instances, the effects of smoking status on metabolism of psychoactive drugs may be an additional way in which smoking could influence measures of brain function in some psychiatric populations.

\section{Importance of smoking as a potential confound for neurobiological findings in psychiatric illnesses}

The importance of controlling for smoking as a potential confound in studies examining neural mechanisms of psychiatric illness is well illustrated by 2 recent studies. In a study including 112 patients with schizophrenia and 77 nonsmoking healthy controls, Schneider and colleagues ${ }^{31}$ showed lower hippocampal and dorsolateral prefrontal brain volumes on MRI in smoking patients compared with nonsmoking patients, and only in smoking patients (not in nonsmoking patients) versus the nonsmoking controls. Adding even more to the complexity is a recent study indicating that smoking may be associated with differential effects on brain structure in patients versus healthy controls. Jørgensen and colleagues ${ }^{32}$ included smokers and nonsmokers both in their sample of 506 patients with severe mental illness (psychotic disorder or bipolar disorder) and in their sample of 237 controls. They found decreased cortical thickness in prefrontal and insular regions in smoking patients compared with nonsmoking patients, but not in healthy smokers compared with healthy nonsmokers. This raises the possibility that patients might be more vulnerable than healthy controls to effects of smoking on brain structure, although the authors were cautious in endorsing this interpretation, as other factors, such as smoking intensity and age, might have accounted in part for these differential effects. The notion that smoking could have significant effects on brain structure or function only in individuals with specific psychiatric disorders but not in healthy controls is an area requiring further investigation.

\section{Smoking cessation among individuals with psychiatric illnesses}

It would be remiss to end an editorial on smoking and psychiatric illness without emphasizing the importance of promoting smoking cessation among individuals with psychiatric illness. It has been estimated that approximately $40 \%$ of premature deaths in the United States caused by smoking occur in individuals with a mental illness. ${ }^{2}$ In addition to effects on general health, smoking cessation may positively impact psychiatric symptomatology. For example, a recent metaanalysis found reductions in measures of anxiety and depression after smoking cessation, with an effect size at least as great as that for antidepressant treatments and a similar effect size in both the general population and populations with psychiatric disorders. ${ }^{33}$ Yet the steady decrease in prevalence of smoking among the general population in recent decades has not been observed in populations with psychiatric disorders. ${ }^{2}$ 
Although the reasons for this are undoubtedly complex, a recent systematic review and meta-analysis found that a large proportion of mental professionals hold a negative attitude toward smoking cessation among psychiatric patients, believing that "patients are not interested in quitting" or "quitting smoking is too much for patients to take on," and indicating a lack of training and time to help patients with their smoking reduction efforts. ${ }^{34}$ This is undoubtedly an area that would benefit from further attention.

Affiliation: Douglas Mental Health University Institute, Department of Psychiatry, McGill University, Montreal, Que., Canada.

Competing interests: None declared.

\section{References}

1. Ng M, Freeman MK, Fleming TD, et al. Smoking prevalence and cigarette consumption in 187 countries, 1980-2012. JAMA 2014; 311:183-92.

2. Prochaska JJ, Das S, Young-Wolff KC. Smoking, mental illness, and public health. Annu Rev Public Health 2016;031816-044618.

3. Pal A, Balhara YP. A review of impact of tobacco use on patients with co-occurring psychiatric disorders. Tob Use Insights 2016;9:7-12.

4. Solmi M, Veronese N, Sergi G, et al. The association between smoking prevalence and eating disorders: a systematic review and meta-analysis. Addiction 2016;111:1914-22.

5. Sciberras E, Mulraney M, Silva D, et al. Prenatal risk factors and the etiology of ADHD-review of existing evidence. Curr Psychiatry Rep 2017;19:1.

6. Thakur GA, Sengupta SM, Grizenko N, et al. Maternal smoking during pregnancy and ADHD: a comprehensive clinical and neurocognitive characterization. Nicotine Tob Res 2013;15:149-57.

7. Ekblad M, Korkeila J, Lehtonen L. Smoking during pregnancy affects foetal brain development. Acta Paediatr 2015;104:12-8.

8. Holz NE, Boecker R, Baumeister S, et al. Effect of prenatal exposure to tobacco smoke on inhibitory control: neuroimaging results from a 25-year prospective study. JAMA Psychiatry 2014;71:786-96.

9. Müller KU, Mennigen E, Ripke S, et al. Altered reward processing in adolescents with prenatal exposure to maternal cigarette smoking. JAMA Psychiatry 2013;70:847-56.

10. Beydoun MA, Beydoun HA, Gamaldo AA, et al. Epidemiologic studies of modifiable factors associated with cognition and dementia: systematic review and meta-analysis. BMC Public Health 2014; 14:643.

11. Durazzo TC, Insel PS, Weiner MW, et al. Greater regional brain atrophy rate in healthy elderly subjects with a history of cigarette smoking. Alzheimers Dement 2012;8:513-9.

12. Teipel S, Grothe MJ; Alzheimer's Disease Neuroimaging Initiative. Association between smoking and cholinergic basal forebrain volume in healthy aging and prodromal and dementia stages of Alzheimer's disease. J Alzheimers Dis 2016;52:1443-51.

13. Kutlu MG, Gould TJ. Nicotine modulation of fear memories and anxiety: Implications for learning and anxiety disorders. Biochem Pharmacol 2015;97:498-511.

14. Slyepchenko A, Brunoni AR, McIntyre RS, et al. The adverse effects of smoking on health outcomes in bipolar disorder: a review and synthesis of biological mechanisms. Curr Mol Med 2016;16:187-205.
15. Levin ED. Complex relationships of nicotinic receptor actions and cognitive functions. Biochem Pharmacol 2013;86:1145-52.

16. Swan GE, Lessov-Schlaggar CN. The effects of tobacco smoke and nicotine on cognition and the brain. Neuropsychol Rev 2007;17: 259-73.

17. Campos MW, Serebrisky D, Castaldelli-Maia JM. Smoking and cognition. [Epub ahead of print]. Curr Drug Abuse Rev 2016 Aug 23.

18. Karama S, Ducharme S, Corley J, et al. Cigarette smoking and thinning of the brain's cortex. Mol Psychiatry 2015;20:778-85.

19. Sutherland MT, Riedel MC, Flannery JS, et al. Chronic cigarette smoking is linked with structural alterations in brain regions showing acute nicotinic drug-induced functional modulations. Behav Brain Funct 2016;12:16.

20. Hanlon CA, Owens MM, Joseph JE, et al. Lower subcortical gray matter volume in both younger smokers and established smokers relative to non-smokers. Addict Biol 2016;21:185-95.

21. Gogliettino AR, Potenza MN, Yip SW. White matter development and tobacco smoking in young adults: a systematic review with recommendations for future research. Drug Alcohol Depend 2016; 162:26-33

22. Weiland BJ, Sabbineni A, Calhoun VD, et al. Reduced executive and default network functional connectivity in cigarette smokers. Hum Brain Mapp 2015;36:872-82.

23. Vergara VM, Liu J, Claus ED, et al. Alterations of resting state functional network connectivity in the brain of nicotine and alcohol users. Neuroimage 2016; pii: S1053-8119(16)30637-1.

24. Weywadt CR, Kiehl KA, Claus ED. Neural correlates of response inhibition in current and former smokers. Behav Brain Res 2017;319: 207-18.

25. Galván A, Schonberg T, Mumford J, et al. Greater risk sensitivity of dorsolateral prefrontal cortex in young smokers than in nonsmokers. Psychopharmacology (Berl) 2013;229:345-55.

26. Lessov-Schlaggar CN, Lepore RL, Kristjansson SD, et al. Functional neuroimaging study in identical twin pairs discordant for regular cigarette smoking. Addict Biol 2013;18:98-108.

27. Desai HD, Seabolt J, Jann MW. Smoking in patients receiving psychotropic medications: a pharmacokinetic perspective. CNS Drugs 2001;15:469-94.

28. Andrade C. Schizophrenia and smoking. J Clin Psychiatry 2012; 73:e725-7.

29. Miksys S, Tyndale RF. Cytochrome P450-mediated drug metabolism in the brain. J Psychiatry Neurosci 2013;38:152-63.

30. Miksys S, Lerman C, Shields PG, et al. Smoking, alcoholism and genetic polymorphisms alter CYP2B6 levels in human brain. Neuropharmacology 2003;45:122-32.

31. Schneider CE, White T, Hass J, et al. Smoking status as a potential confounder in the study of brain structure in schizophrenia. J Psychiatr Res 2014;50:84-91.

32. Jørgensen KN, Skjærvø I, Mørch-Johnsen L, et al. Cigarette smoking is associated with thinner cingulate and insular cortices in patients with severe mental illness. J Psychiatry Neurosci 2015; 40:241-9.

33. Taylor G, McNeill A, Girling A, et al. Change in mental health after smoking cessation: systematic review and meta-analysis. BMJ 2014;348:g1151.

34. Sheals K, Tombor I, McNeill A, et al. A mixed-method systematic review and meta-analysis of mental health professionals' attitudes toward smoking and smoking cessation among people with mental illnesses. Addiction 2016;111:1536-53. 